\title{
Injuries to Ear Ossicles
}

\author{
Anand M Rahalkar* and Mukund D Rahalkar \\ Department of Radiology and Imaging, Sahyadri Hospital, India
}

*Corresponding author: Anand M Rahalkar, Department of Radiology and Imaging, Sahyadri Hospital, India

\begin{abstract}
There are different types of injures to ear ossicles including ossicular chain dislocation, which is separation of the middle ear bones. It results in a hearing loss due to sound not being transmitted properly (conductive hearing loss). Its causes are fracture of one of the bones at the side or base of the skull (temporal bone), a hole in the eardrum that is caused by trauma (tympanic membrane perforation), chronic otitis media or Cholesteatoma.
\end{abstract}

\section{Introduction}

The fractures of temporal bone may be: Longitudinal usually occur with a temporoparietal blow \& cause conductive deafness due to ossicular disruption. SNHL is rare \& can occur due to cochlear concussion. Usually do not cross otic. Transverse typically results from an occipital blow and runs along the length of petrous bone towards petrous apex. It has medial \& lateral subtypes. Medial traverses the fundus of the IAC \& SNHL may be sec. to cochlear nerve transection. Lateral traverses the bony labyrinth \& is often associated with a perilymphatic fistula. Oblique fractures, also called mixed or complex fractures, are the most common types, followed by longitudinal and transverse fractures [1-3]. Ossicular chain dislocation may occur with a temporal bone fracture, traumatic tympanic membrane perforation, head injury or barotrauma. Longitudinal fracture usually occurs with a temporoparietal blow \& causes conductive deafness due to ossicular disruption. SNHL is rare \& can occur due to cochlear concussion. The most common form of ossicular dislocation after temporal bone trauma is separation of the joint connecting the incus to the stapes. The second most common is separation of the joint connecting the malleus to the incus. Fracture of the arch stapes may also occur. The tympanic membrane may or may not be perforated. The causes are external injury to temporal bone, injury by knitting needle, ear bud or pick. The incus is relatively vulnerable to traumatic dislocations (incudostapedial) on account of its weakly anchored position between the firmly attached malleus and stapes. After severe skull trauma also, the incus may suffer dislocation. Malleoincudal dislocation may also occur [4-6]. The incus is relatively vulnerable to traumatic dislocations (incudostapedial) on account of its weakly anchored position between the firmly attached malleus and stapes. After severe skull trauma also, the incus may suffer dislocation. Malleoincudal dislocation may also occur. Complications: perilymphatic fistula, CSF fistula [7], ossicular disruption-commonly incus and Cholesteatoma, $7^{\text {th }}$ nerve paralysis due to fracture extending into facial nerve canal. It may also extend into lachrymal gland. Penetrating traumas through the external ear canal may also cause dislocation of the incus. The incus may remain in the epitympanic recess, dislocate to the most inferior portion of the tympanic cavity or of the external ear canal, or even be destroyed. A thorough evaluation by means of computed tomography in axial and coronal planes of the middle ear and external acoustic meatus is necessary to identify the exact position of the incus in relation to the malleus and the stapes. A unique case of an ossicular chain injury was reported in a young man. Even though the patient's incus was dislocated into the external auditory canal while remaining attached to the stapes, his hearing was not affected and remained nearly normal. Rarely incus has been reported to extend into vestibule, carotid canal or tympanic membrane [2,3]. The causes are external injury to temporal bone, injury by knitting needle, ear bud or pick. Thomsen (3) reported a material of 40 patients with diagnosis of dislocation of the incus is presented. 37 of these patients had either a history of cranial trauma, or information about previous simple mastoidectomy or paracentesis the ossicles are deprived of their essential blood supply and yet nearly unharmed. Nilesh Gupta, Dr. Manish Meshram [4] reported a rare case of dislocation of incus into the external acoustic canal following a fall from stairs with head injury.

CT scan revealed disrupting the Ossicular chain with disarticulation of malleolo-incudal joint and dislocation of incus. A 
bony fragment, giving appearance as shape of incus, noted in the postero-inferior region of right external ear canal. Micro-Otoscopy, 10 days after injury, revealed an intact tympanic membrane covering the fractured - dislocated incus. The fractured segment was displaced and attached to the postero-inferior aspect of the external auditory canal. The other injuries are: malleoincudal joint dislocation, Stapediovestibular dislocation, Stapediovestibular dislocation, Labyrinthine fractures, Dural fistulas, facial paralysis or injury to carotid canal. Tympanic membrane may or may not be perforated. The causes are external injury to temporal bone, injury by knitting needle, ear bud or pick. Treatment in any case is middle ear exploration and ossicular chain reconstruction. This surgery is essentially the same as tympanoplasty; however, there is no hole in the tympanic membrane to fix. Sometimes, the surgery can be performed though the ear canal rather than having to make an incision behind the ear.

\section{Material}

Our hospital is a tertiary care center for head injury. Our experience over a period of 12 years is presented with illustrations.

\section{Illustrations}

a) Case 1: Normal linear and curvilinear structures must not be mistake fractures (pseudo fractures): cochlear \& vestibular aqueducts, sutures, mastoid canaliculus, ITC, singular \& subarcuate canals. These are illustrated in the Figures $1 \mathrm{a}-1 \mathrm{~d}$.

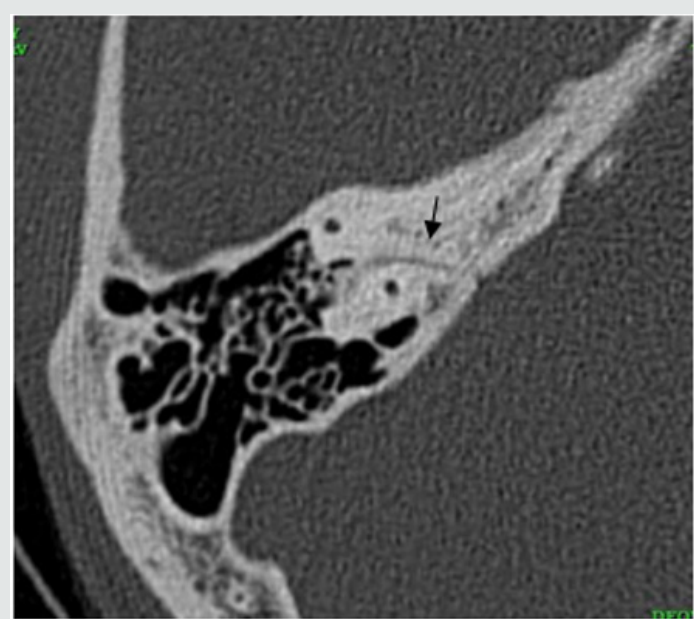

(a)

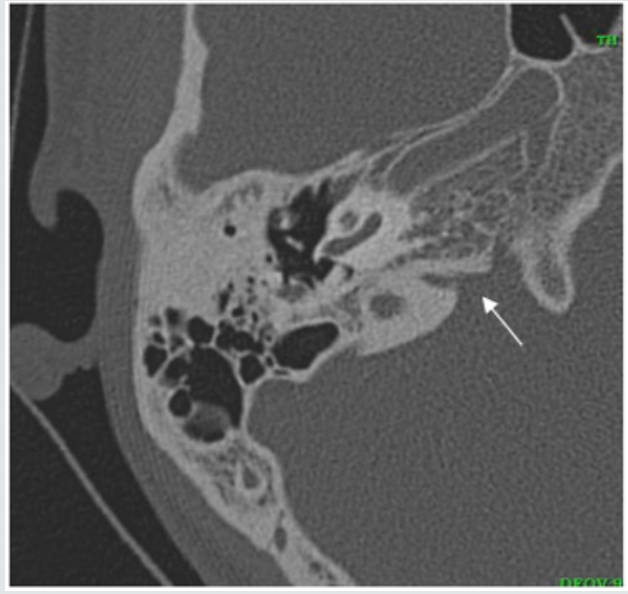

(c)

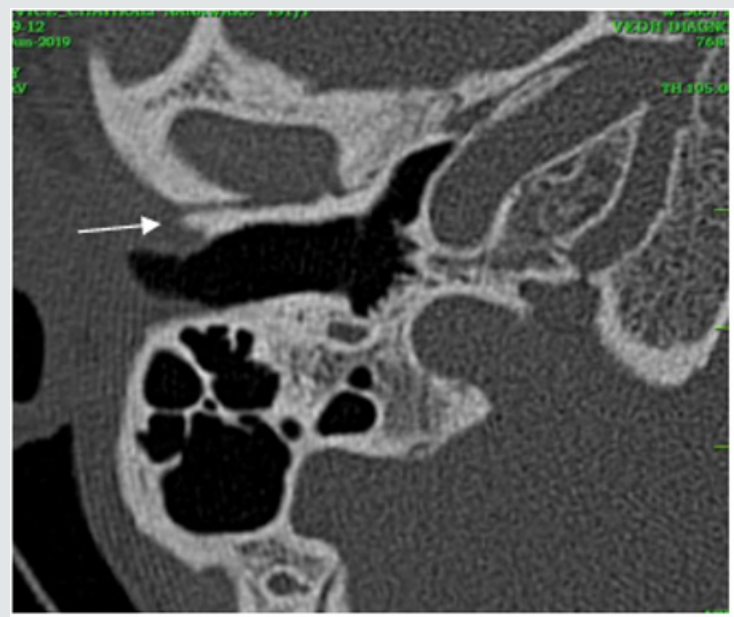

(b)

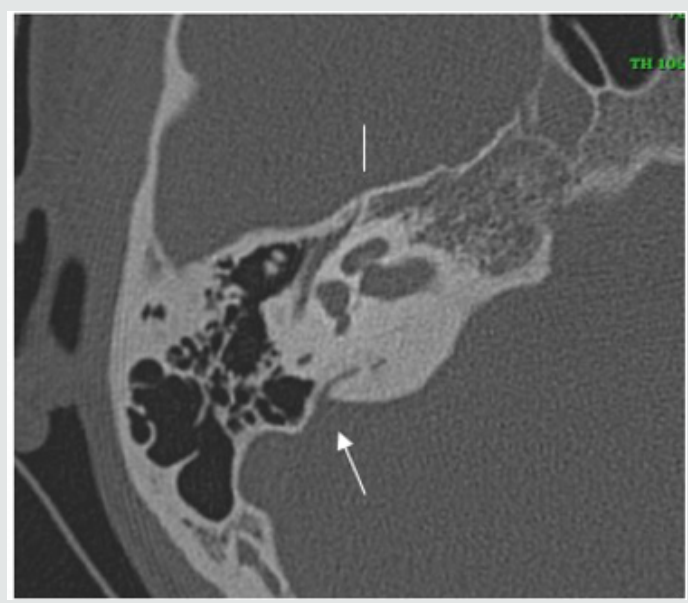

(d)

Figure 1:

a. Subarcuate canal (arrow) carrying nerve and vessel.

b. Superior temporal suture (arrow).

c. Cochlear aqueduct.

d. Vestibular aqueduct (arrow) and glossopharyngeal nerve canal (straight line).

\section{Conclusion}

Images showing pseudo fractures are presented, not to be mistaken for fractures (Figures 2-11). A few typical fractures and complications seen on CT Cisternography are also illustrated, from our experience in nearly 8 years. Dislocation of incus is rare indeed but has been reported with a large in number in some series. 


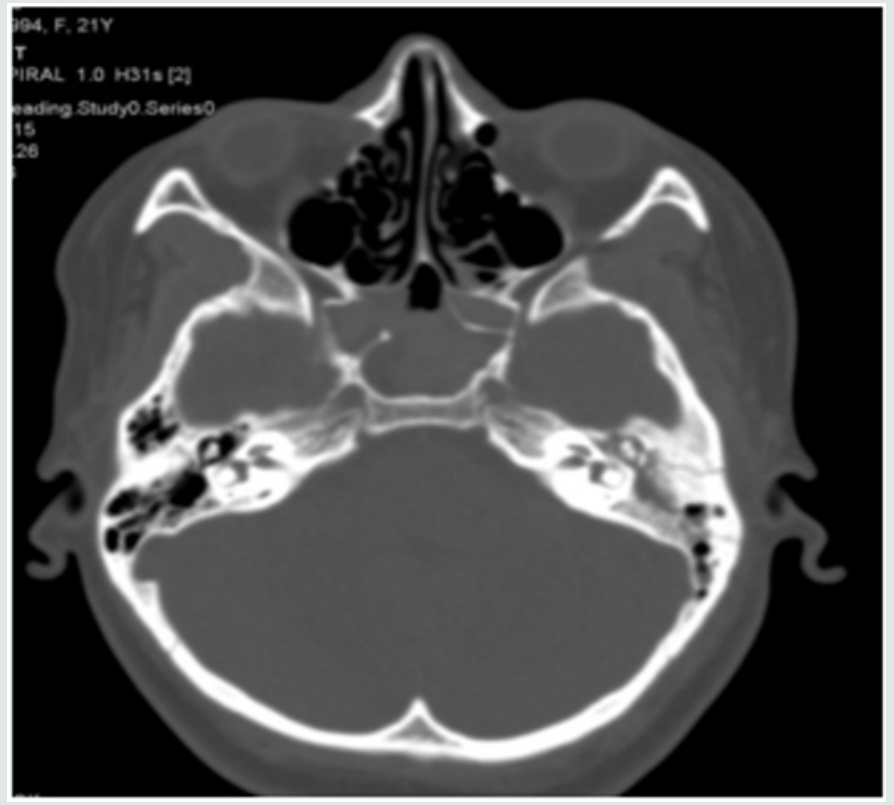

Figure 2: This image reveals a longitudinal fracture of petrous bone on left with hemorrhage in tympanic cavity and includemalleolar dislocation.
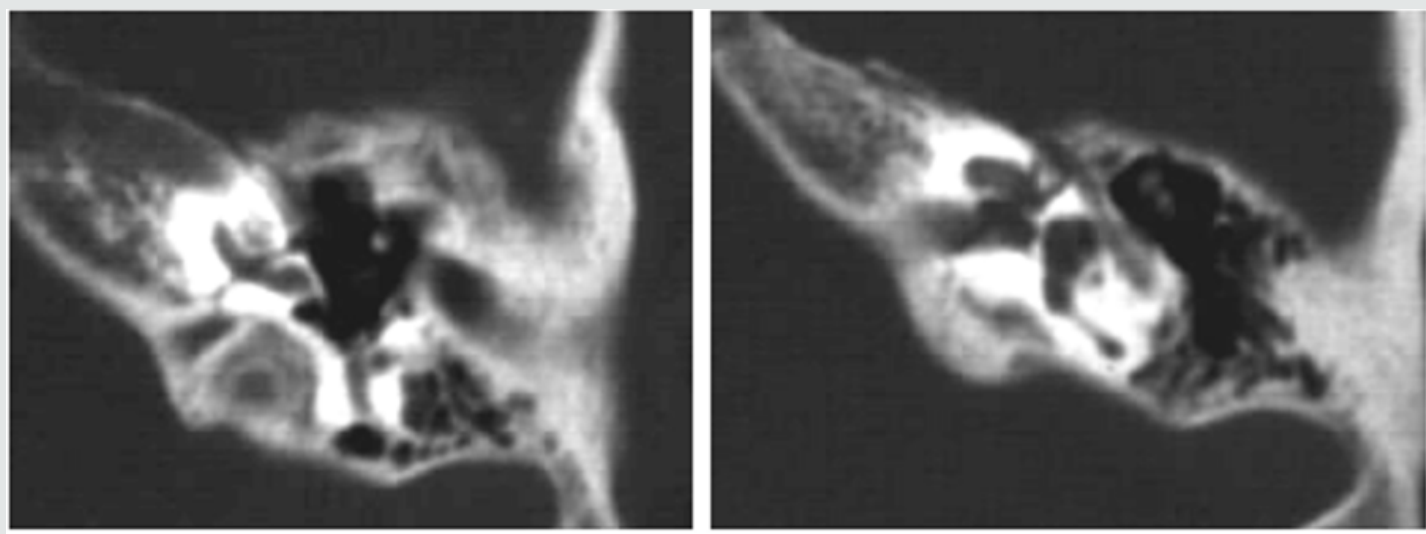

Figure 3: Shows a transverse fracture of petrous bone crossing the turns of vestibule.
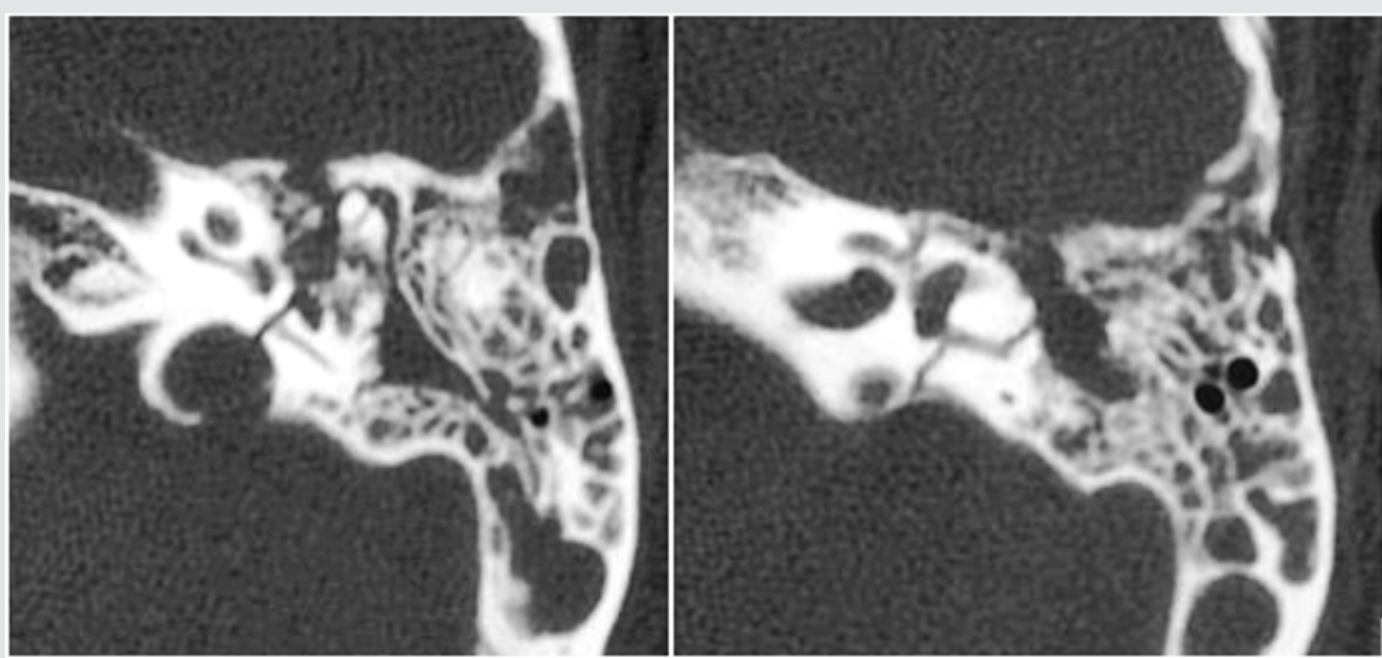

Figure 4: Another case of transverse fracture. A Transverse fracture of petrous bone is seen reaching horizontal semi-circular canal and causing hemotympanum. 


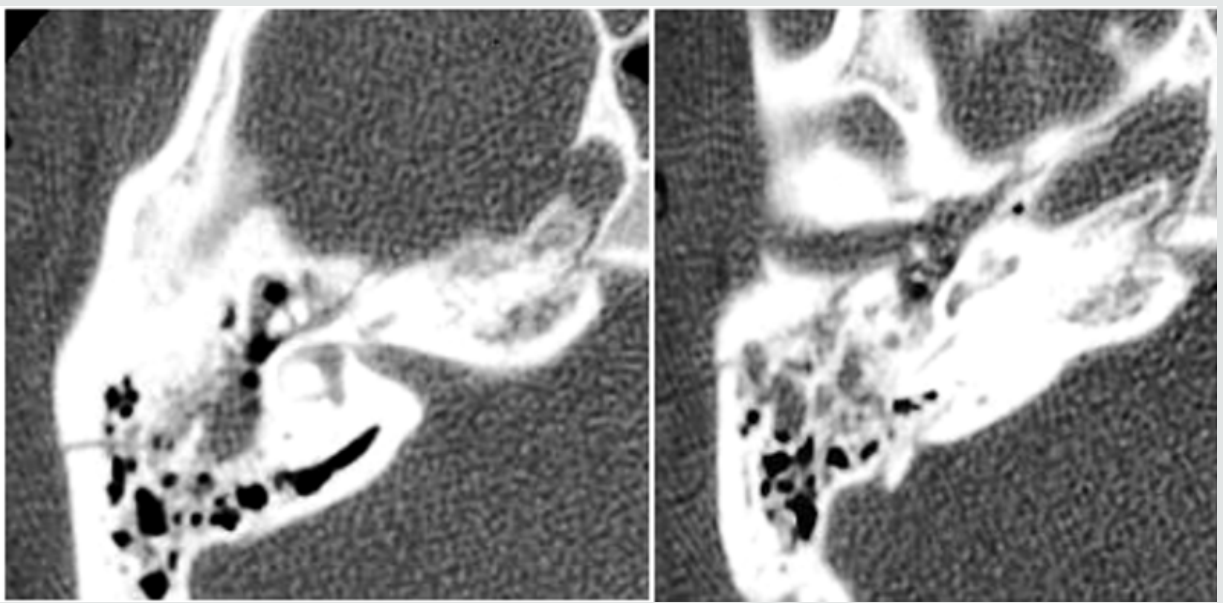

Figure 5: Hemorrhage in external auditory canal and middle ear due to a longitudinal fracture.
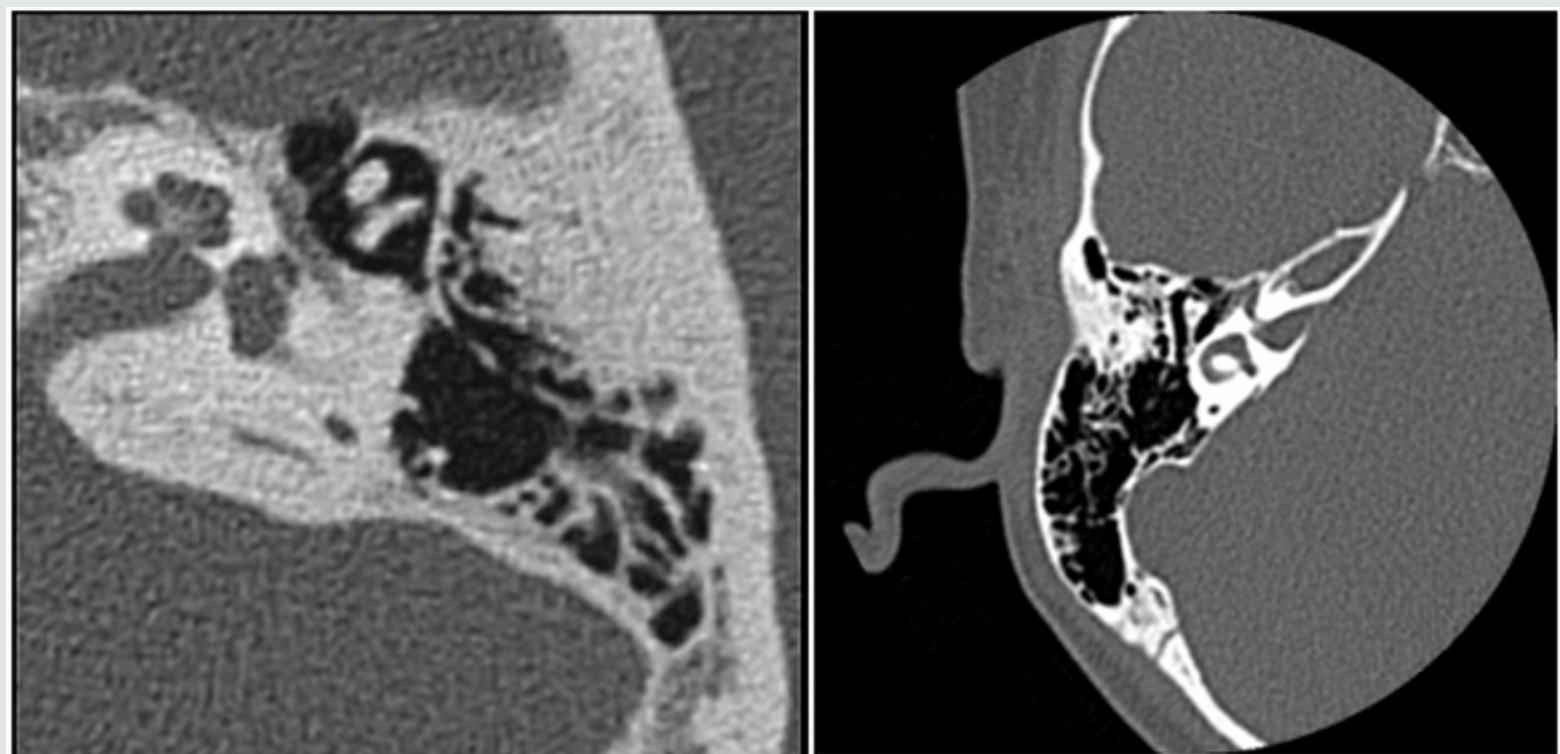

Figure 6: Images of CT show disturbed ice-cream appearance of incus and malleolus.
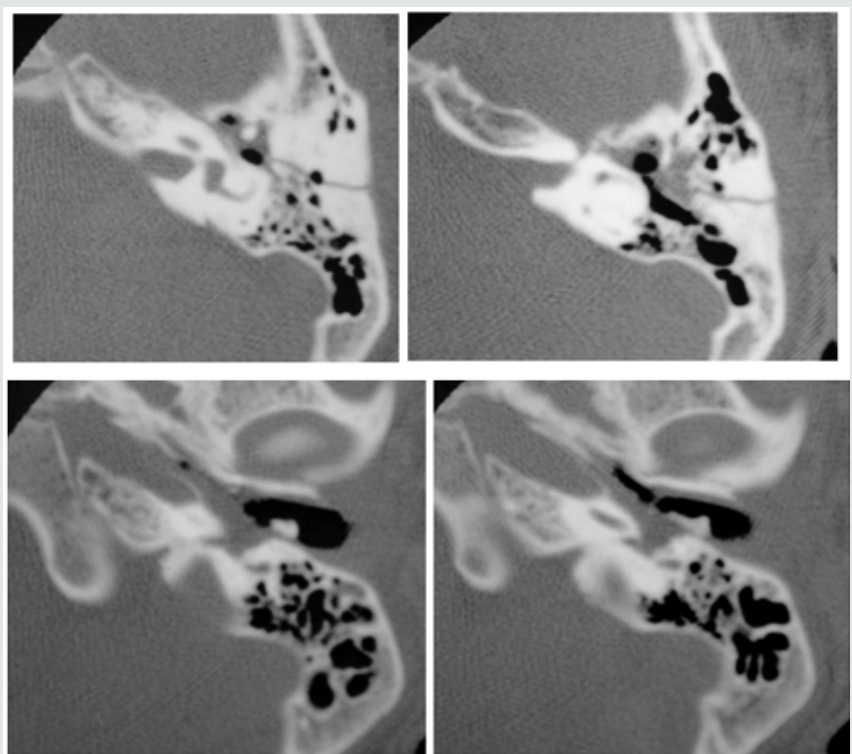

Figure 7: HRCT of mastoid images (upper row axial views and lower row coronal views show incus dislocation (extruded into external auditory meatus. [7] Oculoplastic was carried out. 


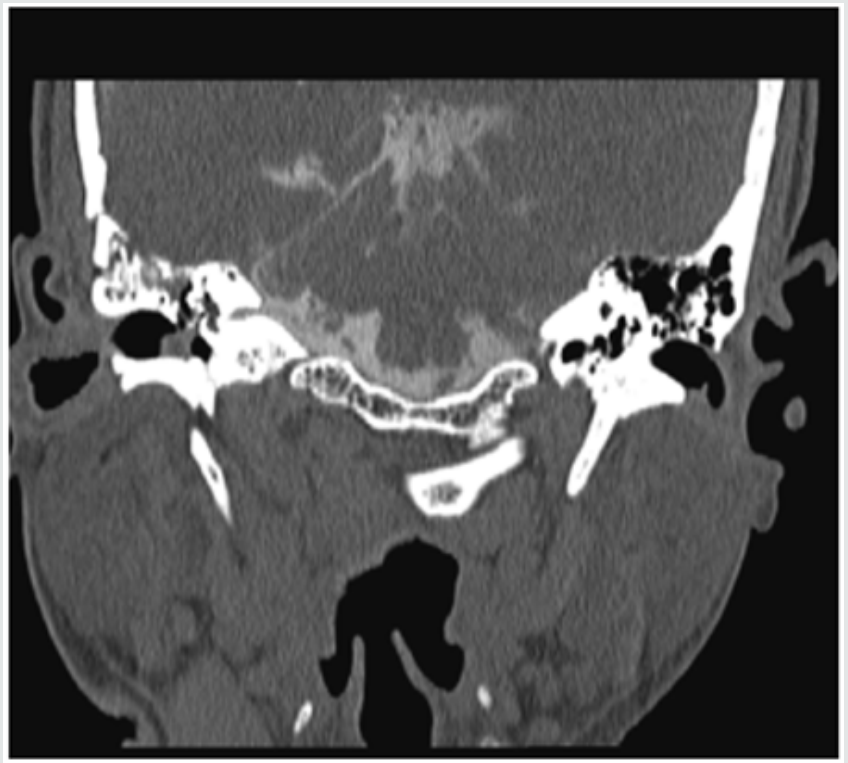

Figure 8: H/o head injury due to Otorrhea due to small leak beneath defect in tegmen tympani on right side.
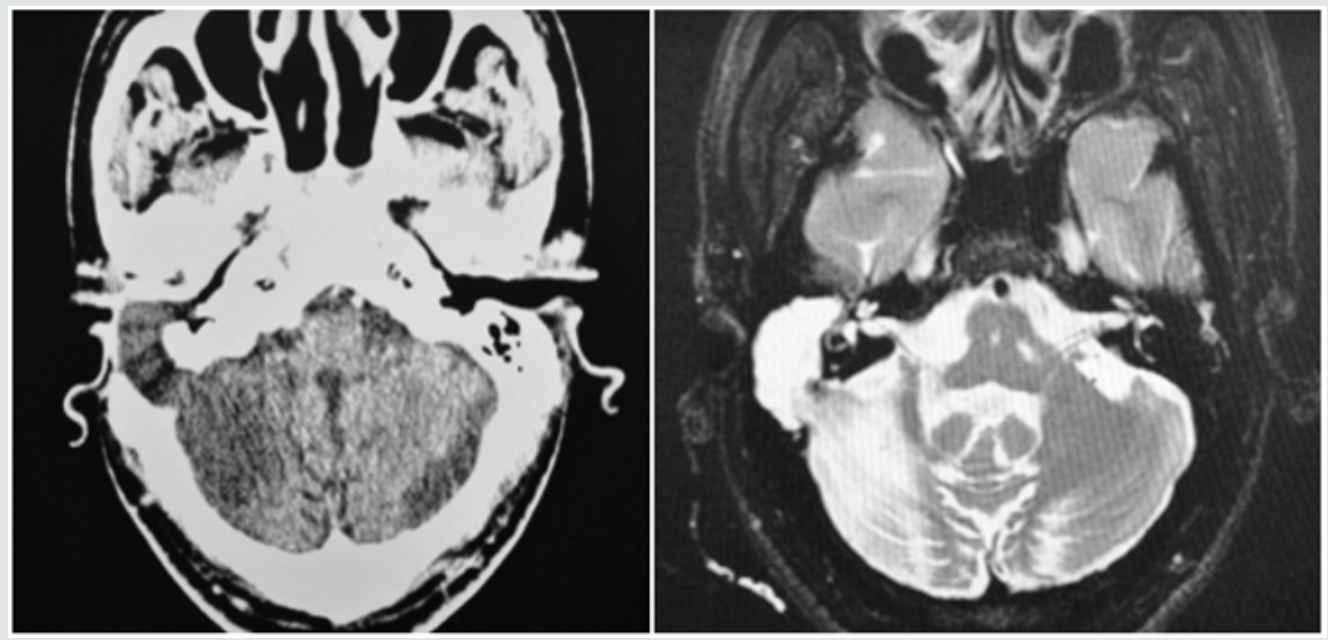

Figure 9: 45-year-old male, $\mathrm{H} / \mathrm{O}$ mastoidectomy in the past. C/O watery discharge from ear. CT reveals a defect in mastoid filled with CSF density lesion. MR confirms.
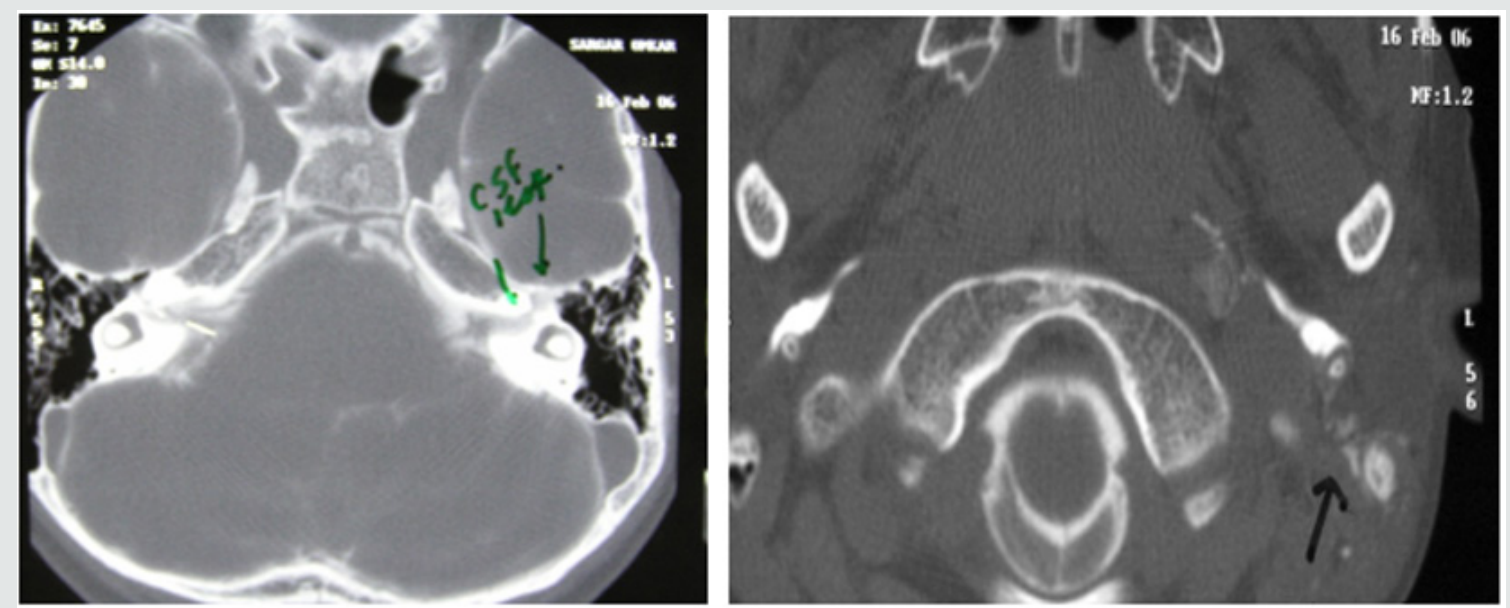

Figure 10: This 11-year-old boy C/O - Repeated attacks of meningitis, operated for CSF fistula. He continues to have attacks of meningitis. Nuclear scan - increased activity in the neck on left. CT cistern gram confirms leakage into genicular fossa and then near the mastoid tip. 


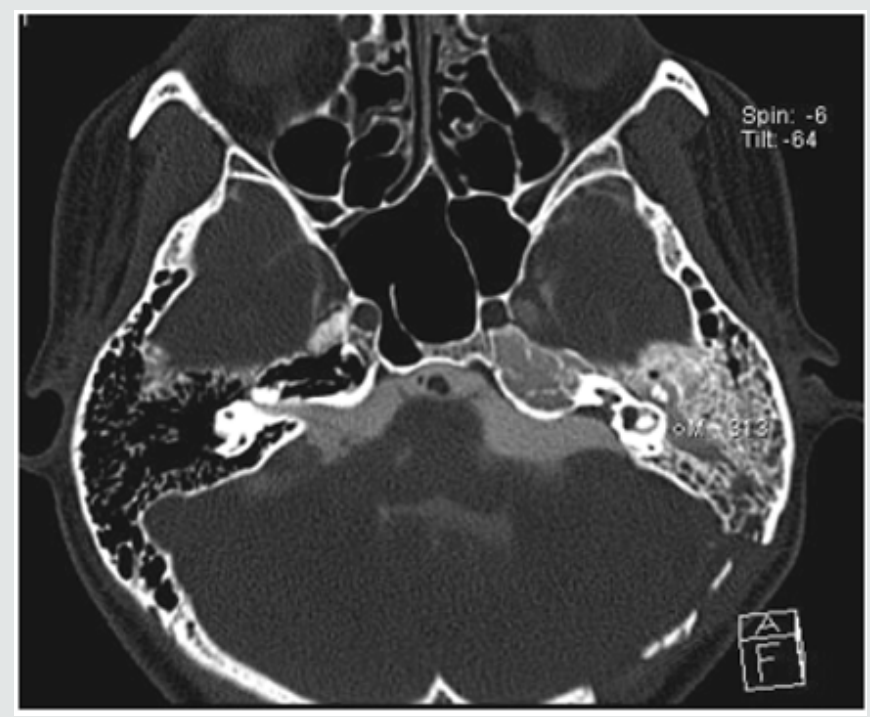

Figure 11: CT cistern gram shows opacification of mastoid cells and antrum as a complication of surgery for acoustic neuroma.

\section{References}

1) Sertac Yetiser, Yusuf Hidir, Hakan Birkent, Satar B, Durmaz A (2008) traumatic ossicular dislocations: etiology and management. American Journal of Otolaryngology (Am J Otolaryngol) 29(1): 31-36.

2) Ana Maria Doffémond Costa, Juliana Oggioni Gaioti, Caroline Laurita Batista Couto, Renata Lopes Furletti Caldeira Diniz, Emilia Guerra Pinto Coelho Motta, et al. (2013) Temporal bone trauma and complications: computed tomography findings. Radiol Bras 146(2).

3) Holzapfel AR, Chang CY, Pereira KD Deguine C, Pulec JL (2005) Ossicular chain dislocation with normal hearing. Ear Nose Throat J 84(6): 351353.

4) Y Tanaka, Hiromi Kojima, Hiroshi Moriyama (2010) Dislocation of the incus into the subcutis of the external auditory canal in a case of temporal bone fracture. Oto-Rhino-Laryngology Tokyo 53(1): 17-21.
5) Nilesh Gupta, Manish Meshram (1995) Post-Traumatic dislocation of the incus into the external acoustic canal: a rare case report. Ear Nose Throat J 74(12): 800.

6) (2018) Dislocation of the Incus A Clinical Survey with Discussion of Role of Blood Supply Thomsen Arch Klin exp Ohr Nas u Kehlk Heilk 203: 166171.

7) Mukund D Rahalkar, Anand M Rahalkar, Vardhan S Joshi, Kailash V Sant, Kourabhi B Zade (2017) Computed Tomography Cisternography for the Management of Cerebrospinal Fluid Fistulae. Otorhinolaryngologsy Clinics: An International Journal 9(3): 1-127.

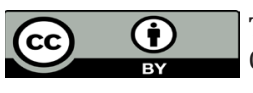

This work is licensed under Creative Commons Attribution 4.0 License

To Submit Your Article Click Here:

Submit Article

DOI: $10.32474 /$ SJO.2019.02.000142

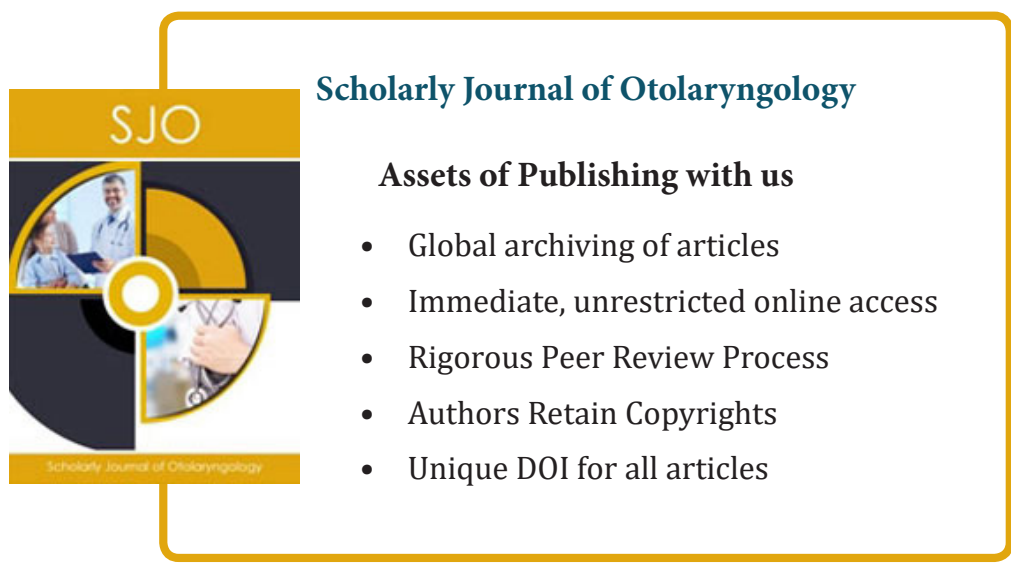

